\title{
Fabrication and characteristics of bismuth oxide based highly nonlinear fiber
}

\author{
Naoki SUGIMOTO ${ }^{\dagger}$ \\ Research Center, Asahi Glass Co., Ltd., 1150, Hazawa-cho, Kanagawa-ku, Yokohama 221-8755
}

\begin{abstract}
Highly nonlinear fiber with step-index type and microstructure type were fabricated using $\mathrm{Bi}_{2} \mathrm{O}_{3}$ based high refractive index glasses. Step-index type fiber with effective core area of $3.3 \mu \mathrm{m}^{2}$ was fabricated using core and cladding glass whose refractive index is 2.22 and 2.13. This fiber exhibits high nonlinearity $\left(\gamma=1300 \mathrm{~W}^{-1} \mathrm{~km}^{-1}\right)$ because of high nonlinearity of the glass material and the small effective core area. The fiber also exhibits low loss $(-1.9 \mathrm{~dB} / \mathrm{m})$ and fusion spliceability to silica fiber. Microstructure type fiber which shows low GVD and high nonlinearity simultaneously was fabricated by a novel inflating method. This fiber has a guiding core structure to reduce the fusion splicing loss when air holes collapse. Wide wavelength tuning range due to small GVD in four wave mixing experiment was demonstrated.

(C)2008 The Ceramic Society of Japan. All rights reserved.
\end{abstract}

Key-words : Nonlinear fiber, Nonlinearity, $\mathrm{Bi}_{2} \mathrm{O}_{3}$

[Received June 12, 2008]

\section{Introduction}

Internet and data traffic continues to grow steadily, which are stimulating the demand for higher information transmission capacity and processing speed of optical network. Optical processing technologies are required from the viewpoint of not only the speed but also 'energy saving' since electrical consumption at network node is now becoming enormous. The optical signal processing technologies will play a key role in future wideband wavelength division multiplexing (WDM) networks and ultrahigh-speed time division multiplexing (TDM) systems. Optical wavelength conversion of WDM signals, optical demultiplexing of TDM signals, and optical signal regeneration are typical examples of such all-optical signal processing. Third-order optical nonlinearity is employed in these applications.

Optical fiber is one of the candidates for third-order nonlinear media because of its high-power density and long interaction length. The conventional silica fibers are widely used as a standard nonlinear medium. However, the nonlinear refractive index of silica based glass is intrinsically low, and thus long fiber length is required to obtain a sufficient nonlinear phase shift. Highly nonlinear fiber allows us to shorten the fiber length and to reduce the required optical power. The nonlinear coefficient $\gamma$ of the fiber is written as

$$
\gamma=2 \pi n_{2} / \lambda A_{\text {eff, }}
$$

where $\mathrm{n}_{2}$ is the nonlinear refractive index, $\lambda$ the wavelength, and $A_{\text {eff }}$ the effective core are of the fiber. ${ }^{1)}$ Thus we have two approaches to enhance the fiber nonlinearity $\gamma$. One is to reduce the effective core area $A_{\text {eff }}$ and the other is to use a glass material whose $\mathrm{n}_{2}$ is high. For example, the $\gamma$ value of a standard single mode silica-based fiber is $2.7 \mathrm{~W}^{-1} \mathrm{~km}^{-1}$, and that of silica-based holey fiber which has very small $A_{\text {eff }}$ is $31 \mathrm{~W}^{-1} \mathrm{~km}^{-1}$. $)$

Several types of glass materials, such as, lead-silicate, chalcogenide glass, tellurite glass systems have been developed to

Corresponding author: N. Sugimoto; E-mail: naoki-sugimoto@ agc.co.jp obtain a high refractive index, ${ }^{3-5)}$ since the third order optical nonlinearity of glass material strongly depends on the linear refractive index (Miller's rule). High fiber nonlinearity can be obtained by applying these high refractive index glasses as nonlinear medium. ${ }^{6,7)}$ Lead silicate based holey fiber was recently reported to show high $\gamma=1820 \mathrm{~W}^{-1} \mathrm{~km}^{-1}$. . Tellurite based fiber whose refractive index is 2.03 was reported to show stimulated Brillouin scattering. ${ }^{9)}$ On the other hand, we have proposed highly nonlinear fiber made up of the $\mathrm{Bi}_{2} \mathrm{O}_{3}$-based glass, and reported that this fiber has a high nonlinear coefficient $\gamma=64$ $\mathrm{W}^{-1} \mathrm{~km}^{-1}$ with the conventional step-index structure and with ordinary $A_{\text {eff }}$ of $20 \mu \mathrm{m}^{2}{ }^{10)}$ This high nonlinearity originates from high nonlinearity of $\mathrm{Bi}_{2} \mathrm{O}_{3}$-based glass. ${ }^{11)}$ This fiber shows relatively low propagation loss less than $-0.8 \mathrm{~dB} / \mathrm{m}$, fusion spliceability to silica fibers, and good mechanical- chemical- and thermal-durability. Owing to these practical characteristics, the $\mathrm{Bi}_{2} \mathrm{O}_{3}$-based glass has also been applied to the highly nonlinear holey fiber. ${ }^{12)}$

In addition to the nonlinearity of fibers, a group velocity dispersion (GVD) of fibers is also important for practical application of nonlinear fibers. High refractive index glasses generally show large dispersion due to electronic transition in UV VIS region. GVD is the summation of material dispersion (DM) and waveguide dispersion (DW), therefore high dispersion of highly nonlinear fibers originates mainly from material dispersion. Microstructure fiber (or Photonic crystal fiber: PCF) is favorable to control waveguide dispersion to reduce the total GVD of fiber. Tellurite based microstructure fiber whose zero dispersion wavelength was shifted to $1.5 \mu \mathrm{m}$ band was reported to show high $\gamma=675 \mathrm{~W}^{-1} \mathrm{~km}^{-1}$ with small $\left.A_{\text {eff }}\left(3.54 \mu \mathrm{m}^{2}\right)^{6}{ }^{6}\right)$

We developed a novel $\mathrm{Bi}_{2} \mathrm{O}_{3}$-based glass material which has high refractive index more than 2.2 at the wavelength of $1.55 \mu \mathrm{m}$. Step-index type fiber was fabricated using this glass as core and a suitable cladding glass to achieve small $A_{\text {eff. }}{ }^{13), 14)}$ Furthermore we developed microstructure type fiber whose GVD is controlled by a novel fabrication method. ${ }^{15)}$ These fibers can be fusion spliced to a silica fiber. In this paper, we report the fabrication of both stepindex type fiber (Bi-NLF) and microstructure type fiber (Bi-PCF) using high refractive index $\mathrm{Bi}_{2} \mathrm{O}_{3}$-based glass. We also report the 
nonlinearity and GVD characteristics of these fibers.

\section{Step index type fiber (Bi-NLF)}

\subsection{Fiber characteristics}

$\mathrm{Bi}_{2} \mathrm{O}_{3}$-based glass was prepared by a conventional melting method. Figure 1 shows wavelength dependence of refractive index and absorption coefficient of $\mathrm{Bi}_{2} \mathrm{O}_{3}$-based glass containing $65.5 \mathrm{~mol} \%$ of $\mathrm{Bi}_{2} \mathrm{O}_{3}$. Refractive index at telecom wavelength $(1.55 \mu \mathrm{m})$ is 2.22 . There is no absorption in telecom wavelength. We fabricated single mode Bi-NLF (cladding diameter of 125 $\mu \mathrm{m})$ with plastic coating. In order to fabricate fiber with small

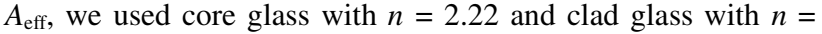
2.13 at $1.55 \mu \mathrm{m}$. Thus the theoretical numerical aperture (NA) of this fiber is calculated to be 0.61 . These glasses have the same thermal properties such as softening point and expansion coefficient as summarized in Table $\mathbf{1}$ and have sufficient thermal stability for fiber drawing. To make $A_{\text {eff }}$ smaller and to satisfy the single mode condition at $1.55 \mu \mathrm{m}$, the core diameter should be ranging from 1.9 to $1.4 \mu \mathrm{m}$. The obtained core diameter is $1.72 \mu \mathrm{m}$ and the fiber diameter is $125.4 \mu \mathrm{m}$. Therefore, the effective core area $A_{\text {eff }}$ is estimated to be $3.3 \mu \mathrm{m}^{2}$. The propagation loss at 1310 $\mathrm{nm}$ was measured to be $-1.9 \mathrm{~dB} / \mathrm{m}$ using a cutback method.

GVD of the fiber was measured by homodyne interferometric method using Agilent 81910A. We estimated that the Bi-NLF has a large normal GVD of $-270 \mathrm{ps} / \mathrm{nm} / \mathrm{km}$ at the wavelength of $1.5 \mu \mathrm{m}$, which is mainly due to the material dispersion of the high refractive index glass.

\subsection{Fusion splicing}

We performed fusion splicing of the Bi-NLF to high-NA silica fibers (HI980, Corning) using a conventional splicing machine. The splicing loss was estimated to be $-9.6 \mathrm{~dB}$. The main cause for this large splice loss is the large MFD mismatch between silica fiber $(6.0 \mu \mathrm{m})$ and Bi-NLF $(2.1 \mu \mathrm{m})$ : We estimated that the loss due to the MFD mismatch is $-4.5 \mathrm{~dB}$ by calculations. In addition, we estimated that $0.5 \mu \mathrm{m}$ misalignment of the axis would result in $-2-\mathrm{dB}$ extra loss. Therefore accurate alignment thorough splicing process is required to achieve low splicing

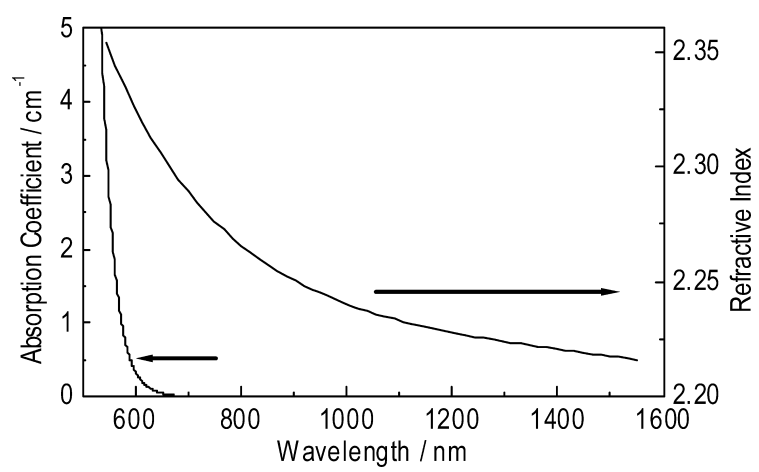

Fig. 1. Refractive index and absorption coefficient of bismuth oxide based glass containing $65.5 \mathrm{~mol} \%$ of $\mathrm{Bi}_{2} \mathrm{O}_{3}$.

Table 1. Refractive Index and Thermal Properties of Core and Cladding Glasses

\begin{tabular}{cccc}
\hline \multicolumn{1}{c}{ Glass } & $n(1550 \mathrm{~nm})$ & $T_{\mathrm{g}}\left({ }^{\circ} \mathrm{C}\right)$ & $\alpha\left(\times 10^{-7} / \mathrm{K}\right)$ \\
\hline Core $\left(\mathrm{Bi}_{2} \mathrm{O}_{3}: 65.5\right)$ & 2.22 & 369 & 113 \\
Cladding $\left(\mathrm{Bi}_{2} \mathrm{O}_{3}: 56\right)$ & 2.13 & 369 & 114 \\
\hline
\end{tabular}

loss. Furthermore, we have succeeded in reducing the fusion splicing loss to less than $-3 \mathrm{~dB}$ using a ultra high NA silica fiber (UHNA4, Nufern) whose NA is 0.35 as an intermediate fiber between SMF28 and Bi-NLF. Schematic diagram of fusion splicing point is shown in Fig. 2. Figure 3 shows loss variation of the splicing when sample number is 20 . Reproducible splicing can be carried out using this method.

\subsection{Four wave mixing experiment}

Figure 4 shows the experimental setup for four wave mixing (FWM). The pump wavelength was fixed to $1550 \mathrm{~nm}$. After being amplified by an Er-doped fiber amplifier, the pump wave was combined with the signal wave whose wavelength was tuned in the range above $1550 \mathrm{~nm}$, and input to the 2-m-long Bi-NLF. From the output spectrum measured by an optical spectrum analyzer as shown in Fig. 5, we can determine the power ratio of

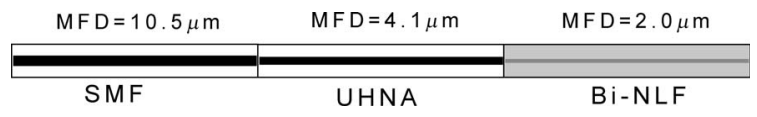

Fig. 2. Schematic diagram of fusion splicing for Bi-NLF using intermediate fiber.

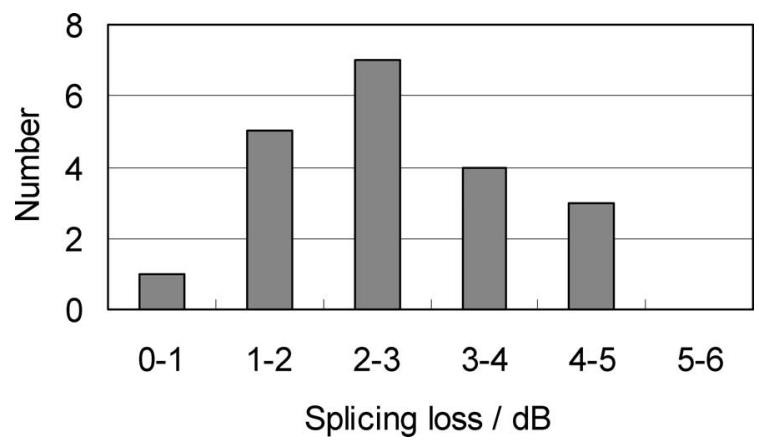

Fig. 3. Splicing loss variation of Bi-NLF.

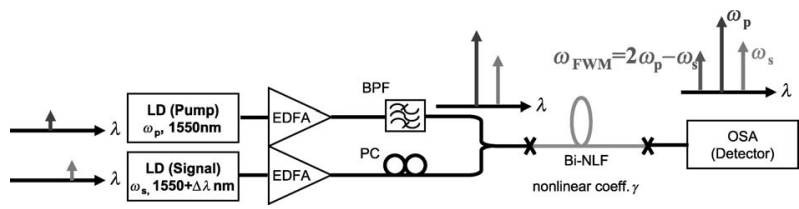

Fig. 4. Experimental setup for four-wave mixing.

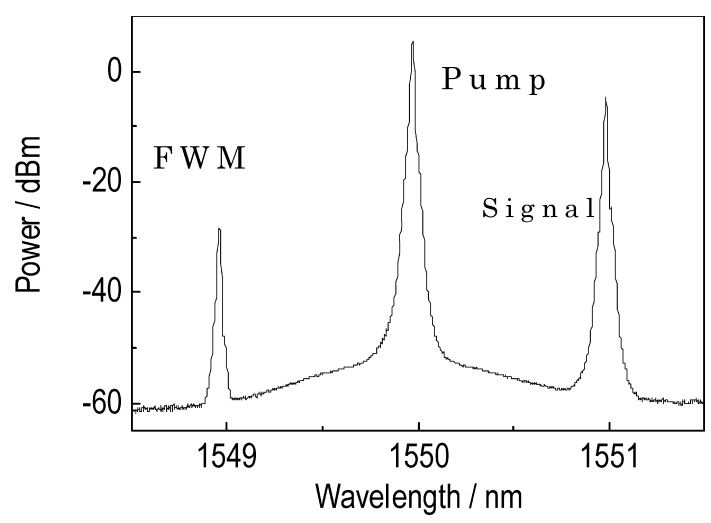

Fig. 5. Output spectrum from Bi-NLF. 
the newly generated FWM wave and the signal wave. The experimental errors of these measurements were less than $0.5 \mathrm{~dB}$. The power ratio $r(z)$ of the idler wave and the signal wave at the propagation distance $\mathrm{z}$ is expressed as

$$
r(z)=\left(\gamma P_{\mathrm{av}} z\right)^{2},
$$

when the signal wavelength is close enough to the pump wavelength. ${ }^{16)}$ In Eq.(2), $\mathrm{P}_{\mathrm{av}}$ denotes the path average power of the pump wave given as

$$
P_{\mathrm{av}}=P_{0}(1-\exp (-\alpha z)) / \alpha z,
$$

where $P_{0}$ is the input power of the pump wave and $\alpha$ the loss constant of the fiber. From Eq.(2), we obtain the nonlinear coefficient $\gamma$ of the Bi-NLF as high as $1300 \pm 160 \mathrm{~W}^{-1} \mathrm{~km}^{-1}$. This value is more than twenty times larger than the reported maximum of the silica-based holey fiber. The nonlinear refractive index $\mathrm{n}_{2}$ calculated from Eq.(1) is $1.1 \times 10-18 \mathrm{~m}^{2} \mathrm{~W}^{-1}$, which is about 40 times larger than that of silica glass and is consistent with the previously reported value. ${ }^{13)}$ Splicing characteristics and the high nonlinearity of the Bi-NLF indicate that the Bi-NLF could be one of the candidates for the practical nonlinear fibers used in optical processing. The change of FWM wave intensity depended on signal wavelength using 70-cm Bi-NLF is shown in Fig. 6. The decrease in the ratio with larger wavelength detuning is due to the high GVD of the fiber. The fitting curve shows the theoretical value estimated using GVD of fiber, and this curve well agrees with the experimental data. This indicates large GVD limits the application of Bi-NLF when multi wavelength waves are used.

\section{Microstructure type fiber (Bi-PCF)}

\subsection{Fabrication of Bi-PCF}

We have developed bismuth-based glasses B036 and B037. These glasses were prepared by a conventional melting method. The refractive indices of B036 and B037 are listed in Table 2. The refractive index depends on $\mathrm{Bi}_{2} \mathrm{O}_{3}$ concentration. The fabricated $\mathrm{Bi}-\mathrm{PCF}$ has core of B037 and cladding of B036 with air holes. The B037 core guides the propagating light to prevent mode leak at the

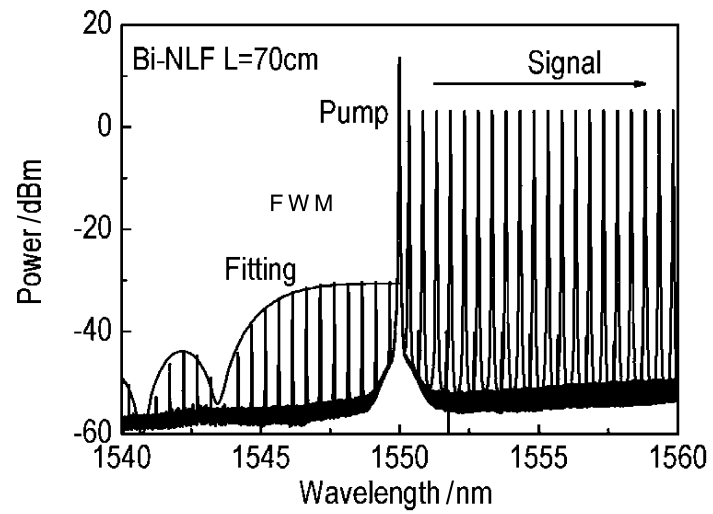

Fig. 6. FWM wave intensity change according to wavelength detuning of signal wave. Fitting curve shows theoretical data estimated using GVD.

Table 2. Refractive Index and Thermal Properties of B037 and B036 Glasses for Bi-PCF

\begin{tabular}{lccc}
\hline \multicolumn{1}{c}{ Glass } & $n(1550 \mathrm{~nm})$ & $T_{\mathrm{g}}\left({ }^{\circ} \mathrm{C}\right)$ & $\alpha\left(\times 10^{-7} / \mathrm{K}\right)$ \\
\hline $\mathrm{B} 037\left(\mathrm{Bi}_{2} \mathrm{O}_{3}: 57\right)$ & 2.139 & 368 & 109 \\
$\mathrm{~B} 036\left(\mathrm{Bi}_{2} \mathrm{O}_{3}: 53.5\right)$ & 2.111 & 370 & 107 \\
\hline
\end{tabular}

fusion splicing point where air holes collapse because of surface tension. In the fabrication process, at first, the fiber preform was fabricated by redrawing the B037 rod and B036 cladding tube. Six holes were drilled in B036 cladding tube as shown in Fig. 7 before redrawing. In redraw process, the glass preform experienced three times of heating cycle, therefore, the thermal properties of these glasses were well adjusted shown in Table 2. The air pressure is applied to inflate the air holes in redraw processes as shown in Fig. 8. ${ }^{15)}$ The SEM image and definition of structural parameters of fabricated Bi-PCF is shown as Figs. 9(a) and (b), and structural parameters are listed in Table 3. It should be noted that the

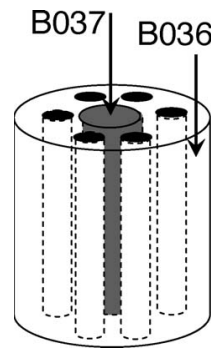

Fig. 7. Schematic structure of Bi-PCF perform composed of guiding core and 6 holes.

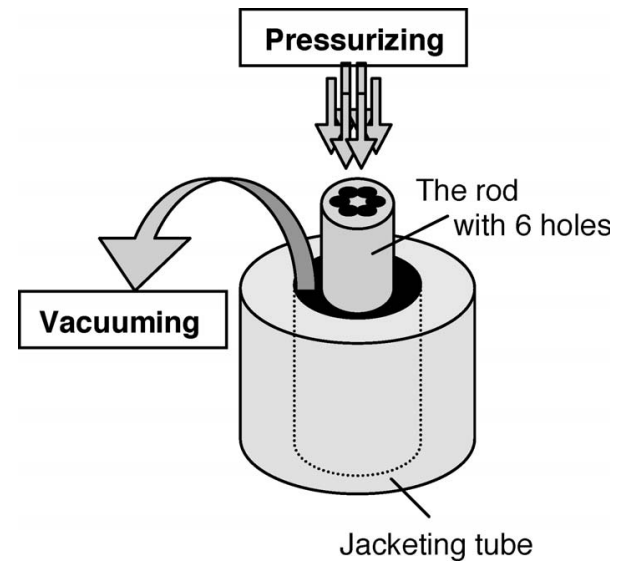

Fig. 8. Schematic diagram of inflating method.
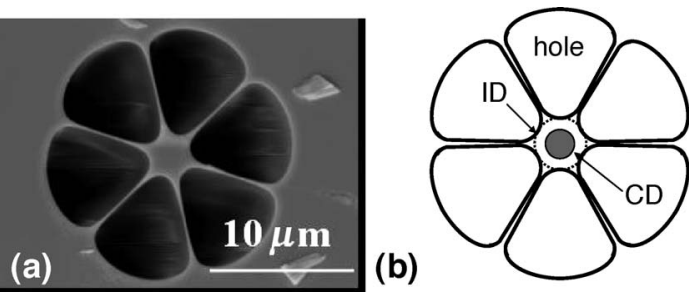

Fig. 9. (a) Cross section SEM image of Bi-PCF. (b) Structural parameters.

Table 3. Obtained Structural Parameters of Bi-PCF along 200-m Length

\begin{tabular}{ccc}
\hline $\begin{array}{c}\text { Core diameter } \\
(\mathrm{CD})\end{array}$ & $\begin{array}{c}\text { Inscribed circle } \\
\text { diameter (ID) }\end{array}$ & $\begin{array}{c}\text { Cladding } \\
\text { diameter }\end{array}$ \\
\hline $1.5-1.6 \mu \mathrm{m}$ & $2.7-2.8 \mu \mathrm{m}$ & $125 \mu \mathrm{m}$ \\
\hline
\end{tabular}


parameters are uniform along 200-m length. This indicates that the inflating method is reproducible fabrication method for microstructure fiber.

We performed a fusion splicing on Bi-PCF fabricated in this work. The best splicing loss $-4.6 \mathrm{~dB} /$ point was obtained and averaged splicing loss is $-6.8 \mathrm{~dB} /$ point. The ideal loss is predicted to be less than $-2 \mathrm{~dB} /$ point if we assume an adiabatic mode conversion. We consider that the large extra splicing loss could attribute to the mode-leak. In fact, we observed hole collapsing at the fusion splicing point, and the length of hole collapsing area $\sim 20 \mu \mathrm{m}$ is too short to make mode-field conversion adiabatic $^{17)}$ even with core-guiding structure.

\subsection{GVD and nonlinearity of Bi-PCF}

The core diameter (CD) of Bi-PCF was designed to have moderate normal dispersion $\sim-25 \mathrm{ps} / \mathrm{nm} / \mathrm{km}$. The full-vector BPM was employed to obtain GVD in microstructure fiber. GVD of Bi-PCF was experimentally obtained by a homodyne interferometric method (Agilent 81910A). In Fig. 10, the experimental result is compared with Bi-NLF of step-index structure (GVD $-270 \mathrm{ps} / \mathrm{nm} / \mathrm{km}$ ). DM of B037 is estimated to be $\sim-170 \mathrm{ps} / \mathrm{nm} /$ $\mathrm{km}$, from the wavelength dependence of refractive index. GVD is obviously well reduced to $-25 \mathrm{ps} / \mathrm{nm} / \mathrm{km}$ by PCF structure. On the other hand, Bi-NLF shows significantly large GVD mainly due to large DM $\sim-210 \mathrm{ps} / \mathrm{nm} / \mathrm{km}$. We measured FWM characteristics of Bi-PCF comparing to Bi-NLF. Figure 11 shows the comparison of FWM intensity between Bi-NLF and Bi-PCF

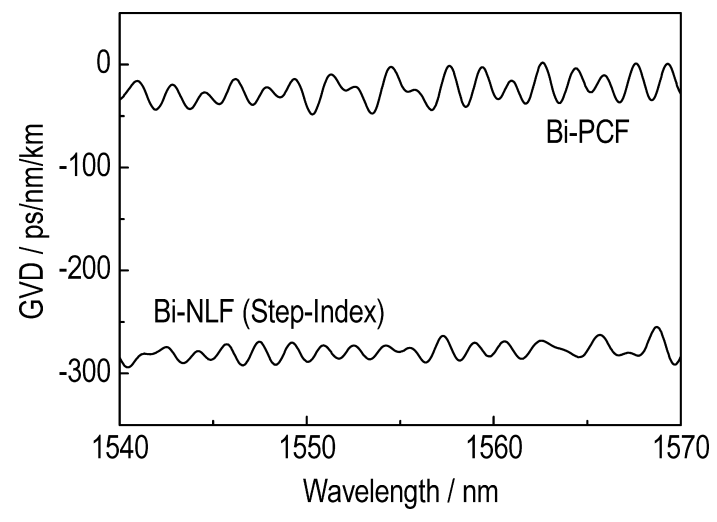

Fig. 10. GVD of Bi-PCF and Bi-NLF.

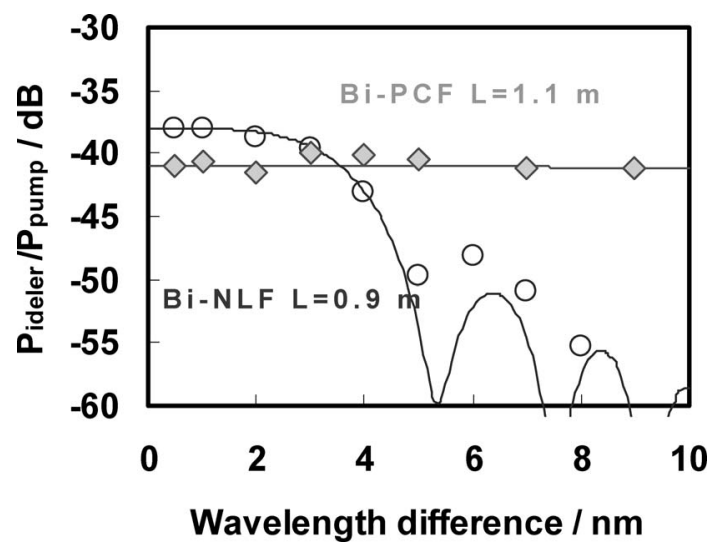

Fig. 11. FWM efficiency of Bi-PCF with wavelength detuning compared with Bi-NLF. Two curves show theoretical data estimated using GVD. when signal wavelength is detuning from the pump wavelength. Two curves shows theoretical value as same in Fig. 6. It should be noted that the significant FWM signal is observed in Bi-PCF even when wavelength difference increases. This wide wavelength tune range is the result of well reduced GVD. From power conversion efficiency, we can calculate the nonlinearity of BiPCF as $\gamma \sim 780 \mathrm{~W}^{-1} \mathrm{~km}^{-1}$. This nonlinearity shows good agreement with the prediction of Miller's rule which describes the relationship between the linear and nonlinear refractive index of glasses. The nonlinearity of the fiber is lower than that of $\mathrm{Bi}_{2} \mathrm{O}_{3}$ glass based holey fiber whose $\gamma$ is $1100 \mathrm{~W}^{-1} \mathrm{~km}^{-1}$ due to small core size. ${ }^{7)}$ This fiber exhibits, however, anomalous dispersion of $+40 \mathrm{ps} / \mathrm{nm} / \mathrm{km}$ at $1550 \mathrm{~nm}$ because of the small core size. The core size in Bi-PCF is designed to achieve smaller normal dispersion of $-25 \mathrm{ps} / \mathrm{nm} / \mathrm{km}$ at $1550 \mathrm{~nm}$ as well as relatively higher nonlinearity.

\section{Conclusion}

We have developed novel $\mathrm{Bi}_{2} \mathrm{O}_{3}$-based glass material whose refractive index $>2.2$ and fabricated conventional step-index type highly nonlinear fiber aiming at applications to all optical signal processing. This fiber exhibits high nonlinearity $(\gamma=1300$ $\mathrm{W}^{-1} \mathrm{~km}^{-1}$ ) because of high nonlinearity of the glass material and the small effective core area. The fiber also exhibits low loss $(-1.9$ $\mathrm{dB} / \mathrm{m})$ and fusion spliceability to silica fiber.

We also fabricated Bi-PCF which shows low GVD and high nonlinearity simultaneously. The guiding core of high refractive index glass B037 enables us fusion splice with low loss of -4.6 $\mathrm{dB}$. We demonstrated wide wavelength tuning range due to small GVD.

Acknowledgement These works were collaborated with the members of Research Center, Asahi Glass Company Ltd.. I would like to express my gratitude to Dr. T. Hasegawa, Mr. T. Nagashima and Mr. S. Ohara.

\section{References}

1) G. P. Agrawal, "Nonlinear Fiber Optics," San Diego, Academic Press (1995).

2) Z. Yusoff, J. H. Lee, W. Belardi, T. M. Monro, P. C. Teh and D. J. Richardson, Opt. Lett., 27, 424 (2002).

3) M. A. Newhouse, D. L. Weidman and D. W. Hall, Opt. Lett., 15,1185 (1990).

4) G. Lenz, J. Zimmermann, T. Katsufuji, M. E. Lines, H. Y. Hwang, S. Spälter, R. E. Slusher, S.-W. Cheong, J. S. Sanghera and I. D. Aggarwal, Opt., Lett., 25, 254 (2000).

5) S. H. Kim, T. Yoko and S. Sakka, J. Am. Ceram. Soc., 76, 2486 (1993).

6) A. Mori, K. Shikano, K. Enbutsu, K. Oikawa, K. Naganuma, M. Kato and S. Aozasa, ECOC2004 Stockholm (2004) Th3.3.6.

7) H. Ebendorff-Heidepriem, P. Petropoulos, S. Asimakis, V. Finazzi, R. C. Moore, K. Frampton, F. Koizumi, D. J. Richardson and T. M. Monro, Optics Express, 12, 5082 (2004).

8) J. Y. Y. Leong, P. Petropoulos, S. Asimakis, H. EbendorffHeidepriem, R. C. Moore, K. Frampton, V. Finazzi, X. Feng, J. H. Price, T. M. Monro and D. J. Richardson, OFC2005 Anaheim (2005) PDP22.

9) G. Qin, H. Sotobayashi, M. Tsuchiya, A. Mori and Y. Ohishi, J. J. Appl. Phys., 46, L810-L812 (2007).

10) K. Kikuchi, K. Taira and N. Sugimoto, OFC2002 Anaheim (2002) ThY6.

11) N. Sugimoto, H. Kanbara, S. Fujiwara, K. Tanaka, Y. Shimizugawa and K. Hirao, J. Opt. Soc. Am. B, 16, 1904-1908 (1999). 
12) H. Ebendorff-Heidepriem, P. Petropoulos, V. Finazzi, K. Frampton, R. C. Moore, D. J. Richardson and T. M. Monro, OFC2004 Los Angels (2004) ThA4.

13) T. Nagashima, T. Hasegawa, S. Ohara, N. Sugimoto, K. Taira and K. Kikuchi, Photonics West 2004 San Jose (2004) 53505357.

14) N. Sugimoto, T. Nagashima, T. Hasegawa, S. Ohara, K. Taira and K. Kikuchi, OFC2004 Los Angeles (2004) PD26.

15) T. Nagashima, T. Hasegawa, S. Ohara and N. Sugimoto, ECOC2006 Canne, We1.3.2.

16) K. Kikuchi and C. Lorattanasane, IEEE Photonics Technol. Lett., 6, 992-994 (1994).

17) J. K. Chandalia, B. J. Eggleton, R. S. Windeler, S. G. Kosinski, X. Liu and C. Xu, IEEE Photon. Technol. Lett., 52 (2001).

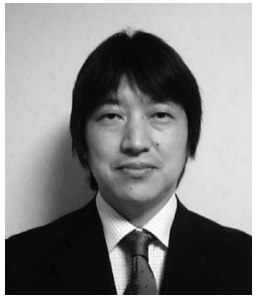

Naoki Sugimoto received the M. S. degree in Molecular Engineering, in 1985 and the Dr. of Engineering degree in 2004, both from Kyoto University, Kyoto, Japan. He joined Research Center, Asahi Glass Co., Ltd., Yokohama, Japan, in 1985, and he is now Relationship Manager of the Research Center. His research interests have included nonlinear optics in fibers and glass materials, amplification characteristics in erbium doped high index fibers, ultrafast processes in glass materials, and glass material design. Dr. Sugimoto is a member of the Ceramic Society of Japan and the Optical Society of America. 